\title{
SNR based Energy Efficient Communication Protocol for Emergency Applications in WBAN
}

\author{
K. Viswavardhan Reddy ${ }^{1}$ \\ Electronics Engineering, Jain University \\ Bengaluru, India
}

\author{
Navin Kumar ${ }^{2}$ \\ Dept. of ECE, Amrita School of Engineering, AVV \\ Bengaluru, India
}

\begin{abstract}
Continuous remote monitoring of a patient's health condition in dynamic environment imposes many challenges. Challenges further get multiplied based on the size of body area sensor network. One such challenge is energy efficiency of sensors. Maintaining longer life of all nodes, especially who participate in communicating vital signals from one network to another towards the base station is very important. In this work, an energy efficient communication protocol for the wireless body area network (WBAN) is proposed. The essential characteristics of the protocol are: random deployment of nodes, formation of clusters, node with high signal to noise ratio (SNR) as cluster head $(\mathrm{CH})$, random rotation of $\mathrm{CHs}$ within each cluster, and so on. The developed algorithm is simulated in MATLAB by varying the number of nodes and networks. Obtained results are compared with some of the recent and most relevant existing works. It is found that there is an enhancement in the network lifetime by $19.5 \%$, throughput by $12.61 \%$ and average remaining energy by $57.21 \%$.
\end{abstract}

Keywords-WBAN; energy efficiency; emergency applications; protocol; remote monitoring

\section{INTRODUCTION}

The usage of information and communication technology (ICT) in healthcare sector is not so widely visible even in the pandemic. Enormous challenges in demand of healthcare centre were encountered [1]. People even in urban areas require doctor appointment and physically visit them if they have any health problem. This has been in practice for a long time and continues even now. Significant research, what we call IOTization, has been around in almost every industry such as smart city, smart home, smart agriculture, smart class room, smart governance, smart and intelligent transport, smart eHealth [2-4]. However, to some extent, smartness is observed in the case of home, in the case of manufacturing but smartness in the case of eHealth is still a miles away. It is limited to a very few individuals who can somehow monitors some of his/her body parameters using smart watch, band, and so on. During this pandemic situation, when social distancing has become very important, health industry should have seen significant growth in terms of usage of monitoring devices and techniques. Unfortunately, we are far behind.

Though, there are many tiny, cheap and smart sensors modules available which can be kept on the body of human within and outside or vicinity of body of human. This type of network is well known as body area network (BAN). Furthermore, this BAN is expected to be dynamic and mobile, such system is known as wireless BAN (WBAN). WBAN allows monitoring of health data of a patient or person from remote. The usage of such networks at this time period could have been significantly increased. Despite that a large number of papers, investigations are available on the subject [5], but practical implementation and usage is not promising due to requirements of bandwidth, high storage capabilities and highpower usage. Additionally, huge information transformation and estimation is required at the edge or cloud. Could computing [6] can be used with regular transformation and data analysis. This inquiry can then be used by the clinicians for providing better treatment of patient in healthcare as well as research. In addition, remote monitoring, consultation, counselling can be easily done. Furthermore, when patient is mobile and dynamic, the system complexity and requirement would be different. One of the important problems would be communicating information from one network to other networks towards the patient's parent cloud, that is, routing of information from the source to destination.

WBAN routing protocols are classified into energy aware routing, cross-layer routing, temperature-based routing, cluster-based routing, posture-based routing and quality of service (QoS)-based routing [7]. An opportunistic powerefficient routing with load balancing (OE2-LB) by eliminating the delay caused during the aggregation process algorithm has been proposed in [8]. It helps in avoiding the loops that occur in routing in a more effective way. Authors claimed to have developed a better algorithm with respect to throughput delay, aggregation time, energy, and live nodes count. A power efficient communication protocol for transmitting the data more reliably is proposed by selecting appropriate next hop node [9]. In this, to select next hop node, a maximum benefit function has been defined. It uses parameters like residual power, bandwidth, efficiency in transmission and number of hops to sink. The performance of the proposed protocol is simulated in MATLAB and evaluated with PERA and NEWATTEMPT protocols. In [10], a clustering routing protocol for WBANs (CRPBA) is developed for maximizing the network lifetime and minimization of power dissipation for the nodes. The performance of the developed algorithm is evaluated with specifications such as total number of nodes 24 ; initial energy of each node is $0.5 \mathrm{~J}$ and total number of 7000 rounds. The reported results showed the first node death at 3375 rounds. In [11], authors developed iMSIMPLE: improved stable increased-throughput multi-hop link adept communication protocol. A cost function is defined by considering various parameters such as distance to sink and residual power has been used for selecting a new forwarding node. Simulations 
were performed with eight nodes having $0.5 \mathrm{j}$ of initial energy and radio parameters like DC current for transmitter and receiver, supply voltage. Results showed that the first node death occurred at 5200 rounds with no mobility. Also, it is learnt that when a patient is static i.e., fixed in location, receive signal strength indicator (RSSI) is normally considered [12]. But when the patient is moving, there will be considerable number of noises as channel keeps changing. Hence, signal to noise ratio (SNR) is more appropriate than the RSSI for variable radio conditions.

Hence in this work, we investigated SNR based power adept communication protocol for not only maximizing the network period by reducing the power dissipation, but also increasing the network throughput and reducing delay in the network. For this, we introduced a weightage function for selecting the cluster head. The weightage function is based on two parameters; high SNR among the nodes and lowest distance from the nodes to cluster head. Also, we compared the performance of our developed algorithm with energy efficient low power robust clustering hierarchy (EELEACH), distributed power efficient clustering (DEEC), threshold sensitive power efficient sensor network protocol (TEEN) and clustering based routing protocol for WBAN (CRPBA).

The contents of this paper have the following details. Section II discusses about related works of various routing protocols with respect to performance metrics, goals, and the cost function that has been developed. Simulation parameters with the radio model and energy model of two modes of communication are discussed in Section III. Section IV deals with proposed algorithm with defined cost function. Finally, results and conclusion were discussed in Sections V and VI, respectively.

\section{RELATED WORK}

Optimized cost effective and energy efficient routing processes (OCER) and Extended-OCER are suggested in [13]. So, as to maximize the network period with the lowest power usage, authors projected a price function with the node's residual energy, path loss, and reliability of link. Nodes owning a less values of price function hold the risks of getting subsequent hop nodes. The functionality of the projected protocol is evaluated using simulations with metrics such as power consumption, throughput and number of packets forwarded. Furthermore, comparison has been made with EPR for indoor medical centres and DMQoS protocols. The outcomes indicate OCER achieves excellent power savings.

When it comes to RK efficient routing protocol [14] has been recommended by the researchers with eight nodes deploying on the human body to keep track of physiological parameters. Nodes are actually split into normal and critical nodes, and that makes use of one hop mechanism and multi hopping respectively. A cost function is identified to pick a forwarder node for multihopping, mostly, based on the least distance to sink and the node's residual power. The functionality of the protocol is then compared and contrasted to ATTEMPT and results suggested that the recommended one outperforms ATTEMPT.
Anwar et. al. developed power aware link effective routing for WBANs (ELR-W) in [15]. Link efficient network model was built to select next hop node with minimum distance to base station and quality link (having very good packet reception ratio). To analyze the efficiency of protocol, parameters such as usage of power, life time of the network, and throughput had been considered for simulations. Very low energy usage, low packet loss i.e., high throughput, and substantial network lifetime were achieved with the ELR-W when in contrast with M-ATTEMPT and iM-SIMPLE.

To have efficient routing approach in WBANs, achieving QoS (throughput, power effectiveness, end-to-end hold off, as well as packet transmission rates) is really very important. As a result, a SDN-enabled and energy-efficient routing algorithm (ESR-W) developed with the usage of the Fuzzy-based Dijkstra method [16] for achieving QoS. So, riverbed modeler simulation software with IEEE 802.15.6 for intra-WBAN, and hubs flow interface protocol for inter-WBAN (SD-WBAN) are used to evaluate the performance of the proposed approach. Simulation results of ESR-W found to be more efficient when compared with AODV, and SDN routing developed for SD-WBAN architecture.

Link-aware and energy efficient pattern for body region networks (LAEEBA) [17] is suggested by the authors to achieve reliable, pathloss efficient and high throughput for WBANs. Besides, on the foundation of higher residual vitality as well as bare minimum distance coming from nodes to BS, a price feature continues to be recommended for choosing the forwarder nodes within the community. The simulations were carried using MATLAB and performance of the LAEEBA is compared with SIMPLE and M-ATTEMPT. A total of 8 nodes with fixed locations, initial energy of $0.5 \mathrm{~J}$ with a range of 10 meters is deployed on the body. The results show that LAEEBA protocol improves the stability of network and network life time with first node dying at 5130 rounds when compared with 2147 and 4436 rounds for M-ATTEMPT and SIMPLE protocols, respectively.

A new routing protocol for heterogenous WBANs named Mobility-supporting adaptive threshold-based thermal-aware energy-efficient multi-hop protocol (M-ATTEMPT) [18] has been proposed by the researchers. Apart from this, a system model is built for placing the nodes on human body. To study this protocol, simulations were carried in MATLAB with 10 nodes as randomly placed, initial energy of these nodes 0.5 joules and their transmission range is 10 meters. Results indicate that first node death occurred at 2700 round and total energy of the network lasted till 3500 rounds. Conclusion: proposed algorithm has consumed very less energy and also achieved very good throughput. The same authors in [12] presented reliability enhanced-adaptive threshold based thermal unaware energy-efficient multi-hop protocol (REATTEMPT) for WBANs. The network life time of ATTEMPT and RE-ATTEMPT lasted for 1450 and 1577 rounds, respectively.

Distance aware relaying energy-efficient protocol (DARE) [19] for enhancing the network life time by reducing the energy consumption has been developed. Simulations performed in MATLAB with 56 sensor nodes, initialized with 
0.3 joule of energy, 8 body relays with 1 joule. The algorithm is compared with M-ATTEMPT and results indicate that in M-ATTEMPT all the 56 nodes are dead after 714 rounds. While, in the case of DARE, for about 1500 rounds all the nodes are dead.

In [20] stable increased-throughput multihop protocol for link efficiency (SIMPLE) is proposed. Similar simulation parameters discussed in [19] were considered and the performance is evaluated based on the metrics like network period, stability lifetime, residual power, throughput, and pathloss [19]. The first node death occurred at 2010 round with ATTEMPT, while, in case of SIMPLE first node death occurred at 4400 rounds. Also, in other performance metrics SIMPLE algorithm outperformed the state of the art.

As we all know, in line of sight (LOS) and non-line of sight (NLOS) conditions, paths between the nodes are experienced with path loss, multipath fading, shadowing effects and noise effects in both. A cooperative LAEEBA (CoLAEEBA) [21] routing scheme has been proposed, considering path loss and collaborative learning. A total of 8 nodes have been considered with three as advanced nodes and remaining five as normal nodes. These normal nodes send the information to advanced nodes. The operation of this protocol is divided into many phases like initialization, co-operation and routing, power consumption, relay selection, and path loss phases. Initial energy of each node is given with $0.3 \mathrm{~J}$ and 0.1 $\mathrm{J}$ for advanced and normal nodes respectively. As we all know that, the performance of the WBANs cab better be understood during simulation studies.

Modified LEACH [22] has been proposed to reduce the energy consumption required for communication. For this, energy and location factors have been considered for electing the $\mathrm{CH}$. The performance evaluation of proposed algorithm, is simulated in MATLAB and then compared with basic LEACH, and LEACH -C. All nodes in LEACH died at 2243, LEACH $-\mathrm{C}$ at 2606 and proposed EE-LEACH at 4958 rounds. Moreover, the proposed protocol i.e., EE-LEACH has balanced the network, with less power consumption, improved data throughput, and prolonged network lifetime.

A single protocol cannot meet the multiple quality of service requirements in WBANs. Moreover, the above protocols are based on cost functions considering single parameters such as calculation of residual energy, minimum distance to local base station, RSSI, load balancing, spatial information, links reliability and path loss. Furthermore, it is observed that few of the above works are carried under ideal conditions and didn't consider any radio models. Thus, in this paper we construct a cost function in selecting optimal cluster head by taking number of parameters such as high SNR, average energy and least distance to $\mathrm{CH}$. With this approach, we not only achieve efficient and reliable communication of data, but also improve network lifetime, and energy efficiency of network.

The developed protocol finds usage in sensor networks. Since, the protocol is energy efficient, it can be used in any sensor networks. Other application supported would be Internet of things (IoT). Some of the applications include minimum energy routine protocol and cluster formation of the networks. There are various power-aware routing protocols including nodes route data destined for the base station particularly through the intermediate nodes. To minimize energy usage, the intermediate nodes are chosen in a way that the transmit amplifier energy is greatly minimized. For example, in clustering, the nodes are organized into clusters that enable communication with a local base station. In turn, the local base stations transmit data to the cellular base stations where accessibility by the end user is made possible. This significantly minimizes the distance nodes that are required to transmit their data since the local base station is closer to other nodes in the cluster.

\section{RAdio SyStem MODEL AND ASSUMPTIONS}

\section{A. Radio Model}

A first order radio model defined in [23] is shown in Fig. 1. In this model, the radio will dissipate: total electronic $\left(\mathrm{T}_{\text {elec }}=\mathrm{E}_{\mathrm{tx}}=\mathrm{E}_{\mathrm{rx}}\right)=50 \mathrm{~nJ} /$ bit to make the transceiver circuitry up and $100 \mathrm{pJ} / \mathrm{bit} / \mathrm{m}^{2}$ for operating transmit power amplifier to achieve desired SNR. Also, we assume that some amount of energy is consumed due to the transmission over channel.

To communicate ' $n$ ' bit message to a distance ' $d$ ' using the assumed model, radio spends:

$E_{t x}(n, d)=E_{t x}(n)+E_{t x-a m p}(n, d)$

$E_{t x}(n, d)=\left(E_{t x}+E_{r x}\right) * n+E_{t x-a m p} * n * d^{2}$

And, while receiving the same message, the radio dissipates

$E_{r x}(n)=t_{\text {power }} * n$

where total power is given as tpower $=\mathrm{E}_{\mathrm{tx}}$ (transmitter power) $+\mathrm{E}_{\mathrm{rx}}$ (receiver power), and $\mathrm{E}_{\mathrm{tx} \text {-amp }}$ is power consumed by the transmitter amplifier electronics. Moreover, we also assumed the amount of energy required for sending a packet from wearable sensor 1 to 2 will be same as that of energy required to for transmitting from node 2 to node 1 at a given SNR.

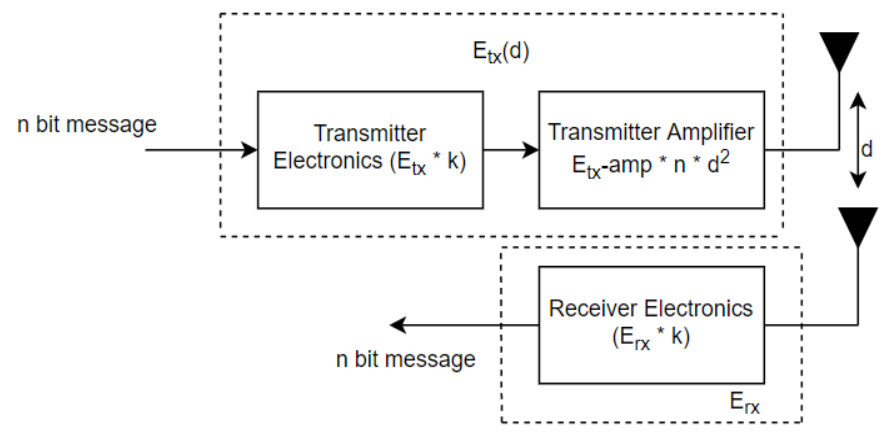

Fig. 1. First Order Radio Model. 


\section{B. Energy for Two Modes of Operation}

Sensor nodes transmit information to local BS in two modes: direct (single hop) and multi hope. In direct mode, nodes send data straight to BS. If BS is too far from them, nodes transmitter will have to spend more energy to transmit to, as ' $d$ ' is greater in eq. 2 . With this, nodes batteries drain faster and network lifetime decreases. To demonstrate the above point, Fig. 2 is considered as a simple linear network, where ' $s$ ' is number of nodes and ' $d$ ' is distance between the nodes.

In direct mode, energy spent for transmitting ' $\mathrm{n}$ ' bit message of a node located at a distance 'sd' from BS is given by:

$E_{\text {direct }}=E_{t x}(n, d=s * d)$

Equation 1 and 2 can be expressed as:

$E_{\text {direct }}=E_{\text {tx }}(n, d=s * d)$

$=T_{\text {elec }} * n+E_{\text {tx-amp }} * n *(s d)^{2}$

$=n\left(T_{\text {elec }}+E_{t x-a m p} s^{2} d^{2}\right.$

In multihop mode, nodes transmit data to BS through intermediate nodes, acting as routers. These intermediate nodes are selected in such way that their transmitter energy is minimized while sending the data. Consider a linear network shown in Fig. 2 with 3 nodes, if a node 1 wants to transmit to node 3 , node 1 should transmit through node 2 only if it satisfies the following condition:

$E_{t x-a m p}\left(n, d=d_{12}\right)+E_{t x-a m p}\left(n, d=d_{23}\right)<$

$E_{\text {tx-amp }}\left(n, d=d_{13}\right)$

where $d_{12}$ is distance from node 1 to node $2, d_{23}$ distance from node 2 to node 3 and $d_{13}$ is distance from node 1 to node 3.

In a multihop mode, nodes near to $\mathrm{BS}$ will receive large amount of data, thus these nodes will die soon. Furthermore, nodes far from BS will have to transmit data with more energy causing these nodes to die and eventually the network goes down. Hence, all the nodes can be arranged into clusters, and communicate with local BSs. Moreover, these local BS transmits data to global BS, comprises of personal computer, phones and other smart electronic items [21], whereas, sensor nodes are all kind of biosensors powered by tiny batteries. Thus, clustering seems to be a solution in providing energy efficient communication between the local BS and global BS. However, the local BS should be given with high energy, otherwise this would die soon.
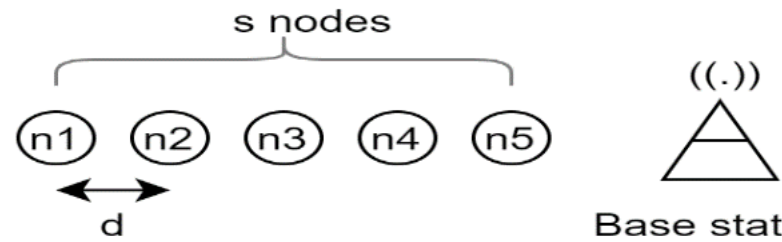

Base station

Fig. 2. Simple Network with Linearity.

\section{Simulation Parameters}

The protocol is based on a model with a person or patient equipped with mMaxBANSize sensor nodes placed outside the body. These nodes will help in monitoring the patient vital signals such as heart rate, body temperature, oxygen levels, blood pressure, blood sugar levels, and respiratory rate, and so on from a remote location. The topology in this network is star with one node acting as a local BS for collecting the data, situated in the centre of the body and others being normal nodes, senses the data and send to local BS in a Multihop or single hop fashion. The reason behind choosing limited number of nodes is due to patient movement in the external environments. All the nodes in the given model are homogeneous and are provided with initial energy $\mathrm{E}_{0}=0.5 \mathrm{~J}$ and considered to have equal computational capabilities. In this work, we assumed a simple first order radio model to run the transmitter and receiver circuitry.

The simulation specifications and radio parameters are shown in Table I.

TABLE I. ShOWS THE SimUlation PARAMETERS

\begin{tabular}{|l|l|}
\hline Parameters & Value \\
\hline Size of BAN (mMaxBANSize) & $6-30$ \\
\hline Network size & $3 \mathrm{mx} 3 \mathrm{~m}$ \\
\hline Sink location & Centre \\
\hline Initial energy ( $\left.\mathrm{E}_{0}\right)$ & $0.5 \mathrm{Joules}$ \\
\hline Number of rounds & 6000 \\
\hline Size of each packet & $4000 \mathrm{bits}$ \\
\hline $\mathrm{E}_{\mathrm{tx}}$ (Transmitter electronics) & $50 \mathrm{~nJ}$ \\
\hline $\mathrm{E}_{\mathrm{rx}}$ (Receiver electronics) & $50 \mathrm{~nJ}$ \\
\hline $\mathrm{E}_{\mathrm{tx}-\text { amp }}$ transmitter amplifier & $100 \mathrm{pJ} / \mathrm{bit} / \mathrm{m}^{2}$ \\
\hline
\end{tabular}

\section{Proposed MEthod}

The protocol is based on communicating the information to $\mathrm{BS}$ by avoiding the battery drainage caused due to direct communication i.e., single hop. Disseminating the data to base station, and achieving the easier convergence of network, sensor nodes themselves organize into clusters with one node being cluster head $(\mathrm{CH})$ or local BS. If we chose $\mathrm{CH}$ before and fixed for the entire system's lifetime, the $\mathrm{CH}$ would die quickly. Thus, there is a necessity to provide random rotation of $\mathrm{CHs}$ among all the nodes within the cluster. Moreover, all the remaining wearable sensors within a cluster will transmit data to $\mathrm{CH}$ to save energy and further $\mathrm{CHs}$ will direct the data to access point by performing data aggregation and segregation. However, the energy consumed by the cluster heads is more when compared with nodes. If it happens continuously, soon the $\mathrm{CHs}$ end up with low energy and thus making network down. Thus, $\mathrm{CH}$ selection is based on the cost criteria given as: 1) by allocating random signal to noise ratio (SNR) among the nodes, and 2) computing the distance between from nodes to $\mathrm{CH}$. If the node is having high SNR and distance between node and $\mathrm{CH}$ is less, then that particular node will become $\mathrm{CH}$ in next iteration. 
In WBAN scenario, the wearable sensors deployed on a body are fixed with respect to their position after the deployment. All these wearables are given initially an equal amount of power except for the local BSs. Initially all the nodes will be in sleep mode, and gets activated only when its allocated time slot is arrived. Once, the nodes get activated, nodes first check its $\mathrm{E}_{\text {total }}$ i.e., total energy. Sensor nodes appoint themselves to be $\mathrm{CH}$ with certain probability. $\mathrm{CH}$ will communicate its status to all wearable sensors in the network. Later nodes in the network identify their neighbors' nodes and forwarder nodes to build a table using eq. (8) and (9).

- Neighbor nodes are identified using:

$N(n)=\{j \mid j \in S, d(i, j)<r\}$

where ' $S$ ' is set of all the sensor nodes, $r$ is the communication radius, $d(i, j)$ is distance from ith node to $j$ th node.

- Forward nodes are identified using:

$F N(i)=\{j \mid j \in N(i), d(j, B S)<d(i, B S)\}$

where $\mathrm{d}(\mathrm{j}, \mathrm{BS})$ is given as distance from $\mathrm{jth}$ node to local BS.

Later, each node will decide to which cluster should belong to by choosing $\mathrm{CH}$ with minimum energy for communication. Finally, $\mathrm{CH}$ will allocate certain schedules to nodes for the transmission of data. With this, node allocated for that particular slot will turn on its radio and transmits the information. Rest all other nodes will be in inactive state.

\section{A. Cluster Head Selection}

- Initially when the clusters formation starts, all the nodes within the cluster can participate in $\mathrm{CH}$ election. $\mathrm{CH}$ election is based on SNR, energy and distance factor of all the nodes.

- Firstly, $\mathrm{CH}$ is elected based on evaluation of SNR randomly for all the nodes using temporary variables and rand function. SNR is generally expressed as:

$S N R=\frac{P_{\text {signal }}}{P_{\text {noise }}}$

- If SNR $\geq$ threshold; only then node will be having higher priority in becoming cluster head. Using this, all the nodes will get an equal opportunity to become $\mathrm{CH}$. Since we have fewer nodes the probability of becoming $\mathrm{CHs}$ is high.

- Each node will have to spend certain amount of energy for data transfer, which is different for every node. Later nodes with maximum energy will be participating in $\mathrm{CH}$ election process using eq. (11), where $\mathrm{E}_{\text {node }}$ is node's energy and $E_{\text {residual }}$ is remaining energy at current round.

$E_{\text {node }}=\frac{E_{\text {residual }}}{E_{\text {total }}}$

- The amount of power dissipated will depend on the distance between source and $\mathrm{CH}$. If distance $\leq$ do (average distance); nodes estimate their distance with respect to $\mathrm{CH}$ and node with minimum distance gets higher probability of becoming next $\mathrm{CH}$. Hence, for the next round, $\mathrm{CH}$ is elected using modified equation given in (12):

$$
\begin{aligned}
& T h=\left[\frac{P}{1-P\left(\operatorname{cr}_{\text {mod }}\left(\frac{1}{P}\right)\right)} *\left(\frac{S^{2} R_{\text {current }}}{S R_{\text {total }}}\right) *\left(\frac{\text { distance }_{\text {current }}}{\text { distance }_{\text {total }}}\right) *\right. \\
& \left.\left(\frac{E_{\text {current }}}{E_{\text {total }}}\right)\right]
\end{aligned}
$$

where ' $p$ ' represents probability that the node has to become cluster head and ' $\mathrm{cr}_{\text {mod }}$ ' is the current round as shown in algorithm 1.

Algorithm1: Energy efficient communication protocol for WBAN applications

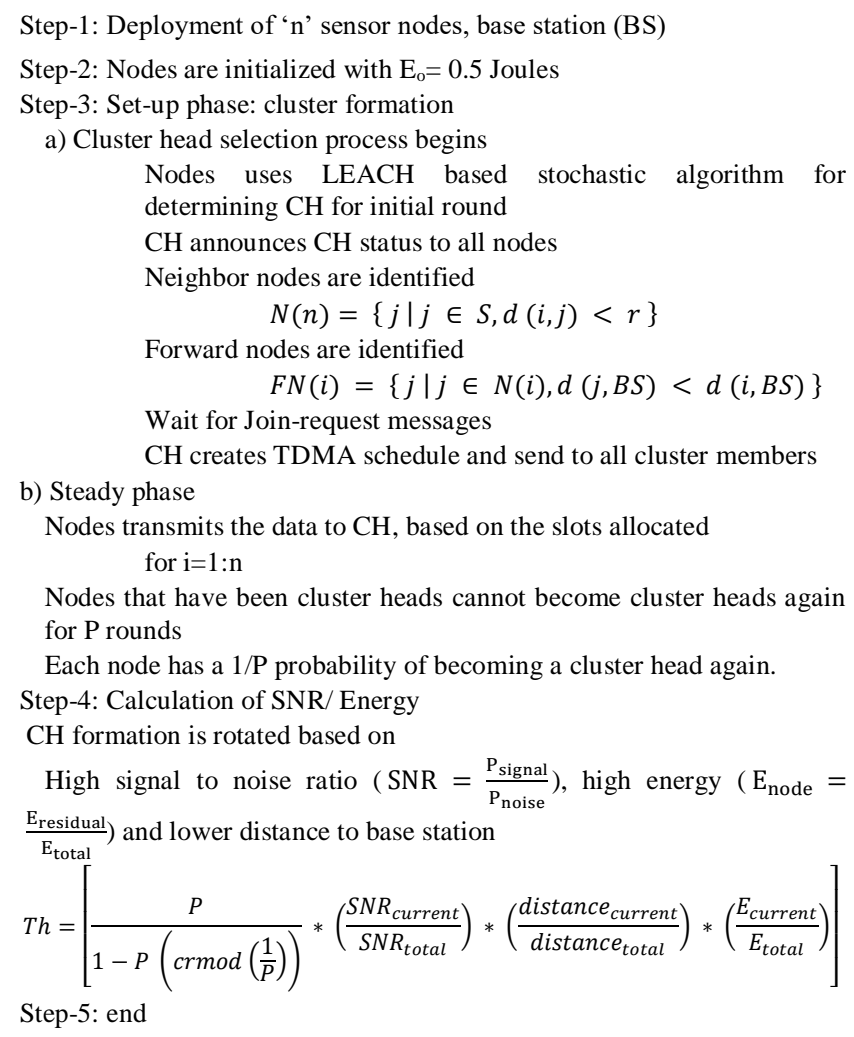

Nodes that have been cluster heads cannot become cluster heads again for $\mathrm{P}$ rounds

Each node has a 1/P probability of becoming a cluster head again.

Step-4: Calculation of SNR/ Energy

$\mathrm{CH}$ formation is rotated based on

High signal to noise ratio $\left(\mathrm{SNR}=\frac{\mathrm{P}_{\text {signal }}}{\mathrm{P}_{\text {noise }}}\right)$, high energy $\left(\mathrm{E}_{\text {node }}=\right.$ $\left.\frac{E_{\text {residual }}}{E_{\text {total }}}\right)$ and lower distance to base station

$T h=\left[\frac{P}{1-P\left(\operatorname{crmod}\left(\frac{1}{P}\right)\right)} *\left(\frac{S N R_{\text {current }}}{S N R_{\text {total }}}\right) *\left(\frac{\text { distance }_{\text {current }}}{\text { distance }_{\text {total }}}\right) *\left(\frac{E_{\text {current }}}{E_{\text {total }}}\right)\right]$

Step-5: end

\section{RESUltS AND DisCUSSION}

Simulations were carried in MATLAB R2019b and performance analysis is compared with various algorithms such as EELEACH, DEEC, TEEN and CRPBA. The following parameters have been considered for performance evaluation: average network life time, throughput, average energy dissipation with varying number of nodes. To have realistic view, we have varied number of nodes between 8 and 30 .

Assuming that in a WBANs patient or person, it can be equipped with 8 or 12 or 20 or 30 nodes. For the given number of nodes each protocol is simulated for five times to find exact accuracy of the algorithm and also average number of rounds with $85 \%$ of the nodes died. 


\section{A. Network Lifetime}

In the first iteration, for 8 nodes, after the simulation starts, $85 \%$ of all the nodes died after 3100 rounds for EELEACH, 3300 for DEEC, 3900 for TEEN, 4170 for CRPBA and proposed algorithm lasts for 5850 rounds. Similarly, iteration 2, 3, 4, and 5 are carried and their values are shown in Table II. Again, an average has been taken among the results obtained, to get more accurate results (total values of each protocol divided by 5). Finally, for 8 nodes we found that our algorithm has performed $23.58 \%$ better than other algorithms. Similarly, for 12 nodes, the average lifetime values are 2159 , 2551, 3526, 4060 and 5059 for EELEACH, DEEC, TEEN, CRPBA and Proposed protocol respectively. Later for 12 nodes we found that our algorithm has performed $19.75 \%$ better than other algorithms.
Similarly, for 20 nodes, the average lifetime values are 1669, 2055, 2623, 3431 and 4065 for EELEACH, DEEC, TEEN, CRPBA and Proposed protocol, respectively.

Later for 20 nodes we found that our algorithm has performed $16.05 \%$ better than other algorithms. Similarly, for 30 nodes, the average lifetime values are 1707, 1880, 2068, 2812 and 3377 for EELEACH, DEEC, TEEN, CRPBA and Proposed protocol respectively. Later for 30 nodes we found that our algorithm has performed $16.7 \%$ better than other algorithms. From Fig. 3 it is can be observed that; the network period is decreasing as the number of nodes are increasing. Hence, an optimal number of nodes that can be placed on body are 8 to have very good network lifetime. Reasons: Due to the interference caused by nodes, packet loss, delay, retransmissions increase. With these factors, power of the nodes disseminates faster.

TABLE II. Performance Evaluation of Network Lifetime For VARIOUS Protocols With 8, 12, 20 AND 30 Nodes

\begin{tabular}{|c|c|c|c|c|c|c|}
\hline \multirow[t]{2}{*}{ Nodes } & \multicolumn{5}{|c|}{ Network Life Values for five simulations } & \multirow[t]{2}{*}{ Analysis } \\
\hline & EELEACH & DEEC & TEEN & CRPBA & Proposed & \\
\hline \multirow{5}{*}{8} & 3100 & 3300 & 3900 & 4170 & $\mathbf{5 8 5 0}$ & \multirow{6}{*}{$\begin{array}{l}\text { Proposed vs Best } 1-(4307 / 5636)=\mathbf{2 3 . 5 8 \%} \\
\text { Better }\end{array}$} \\
\hline & 3355 & 3775 & 3875 & 4335 & 5620 & \\
\hline & 3200 & 3500 & 3572 & 4224 & 5575 & \\
\hline & 3757 & 3558 & 3550 & 4550 & 5380 & \\
\hline & 3250 & 3575 & 3680 & 4255 & 5755 & \\
\hline Avg Net Life & 3332 & 3542 & 3715 & 4307 & 5636 & \\
\hline \multirow{5}{*}{12} & 2112 & 2673 & 3500 & 3878 & 5200 & \multirow{6}{*}{$\begin{array}{l}\text { Proposed vs Best } 1-(4060 / 5059)=\mathbf{1 9 . 7 5 \%} \\
\text { Better }\end{array}$} \\
\hline & 2220 & 2527 & 3425 & 4258 & 5075 & \\
\hline & 2001 & 2580 & 3444 & 3955 & 4836 & \\
\hline & 2110 & 2422 & 3705 & 4220 & 5160 & \\
\hline & 2350 & 2555 & 3556 & 3988 & 5025 & \\
\hline Avg Net Life & 2159 & 2551 & 3526 & 4060 & 5059 & \\
\hline \multirow{5}{*}{20} & 1544 & 1919 & 2545 & 3096 & 4115 & \multirow{6}{*}{$\begin{array}{l}\text { Proposed vs Best } 1-(3431 / 4065)=\mathbf{1 6 . 0 5 \%} \\
\text { Better }\end{array}$} \\
\hline & 1725 & 2215 & 2617 & 3211 & 4256 & \\
\hline & 1780 & 2007 & 2528 & 3857 & 3900 & \\
\hline & 1698 & 2058 & 2770 & 3990 & 4077 & \\
\hline & 1599 & 2075 & 2657 & 3004 & 3975 & \\
\hline Avg Net Life & 1669 & 2055 & 2623 & 3431 & 4065 & \\
\hline \multirow{5}{*}{30} & 1705 & 1902 & 2002 & 2770 & 3452 & \multirow{6}{*}{$\begin{array}{l}\text { Proposed vs Best } 1-(2812 / 3377)=\mathbf{1 6 . 7 \%} \\
\text { Better }\end{array}$} \\
\hline & 1778 & 1885 & 2089 & 2688 & 3351 & \\
\hline & 1658 & 1850 & 2180 & 2910 & 3317 & \\
\hline & 1595 & 1897 & 1998 & 2805 & 3477 & \\
\hline & 1801 & 1867 & 2070 & 2888 & 3288 & \\
\hline Avg Net Life & 1707 & 1880 & 2068 & 2812 & 3377 & \\
\hline
\end{tabular}




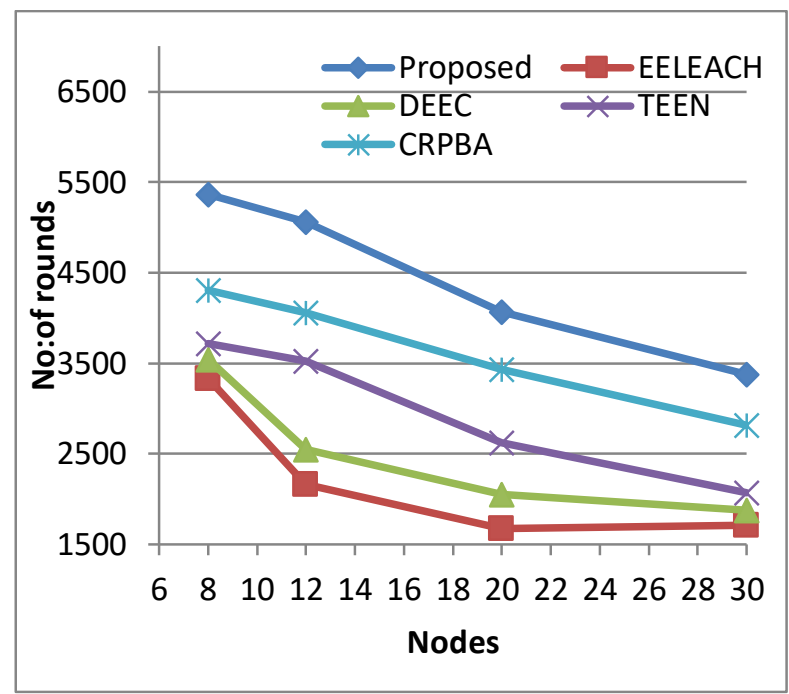

Fig. 3. Number of Rounds over Number of Nodes.

\section{B. Average Remaining Energy Per Node after $85 \%$ of Nodes Died}

From Fig. 4 and Table III, it is seen that the amount of power dissipated in the network is decreasing as the number of nodes are increasing. For EELEACH, DEEC and TEEN protocols, the even after $85 \%$ of the node's death, the amount of energy is decreasing gradually which will lead to network down, whereas in the case of CRPBA and proposed protocol, the curve is flat and leading to efficient network. Hence, an optimal number of nodes that can be placed on body are 8 to have very good network lifetime. Reasons: Due to the interference caused by nodes, packet loss, delay, retransmissions increase. With these factors, power of the nodes disseminates faster.

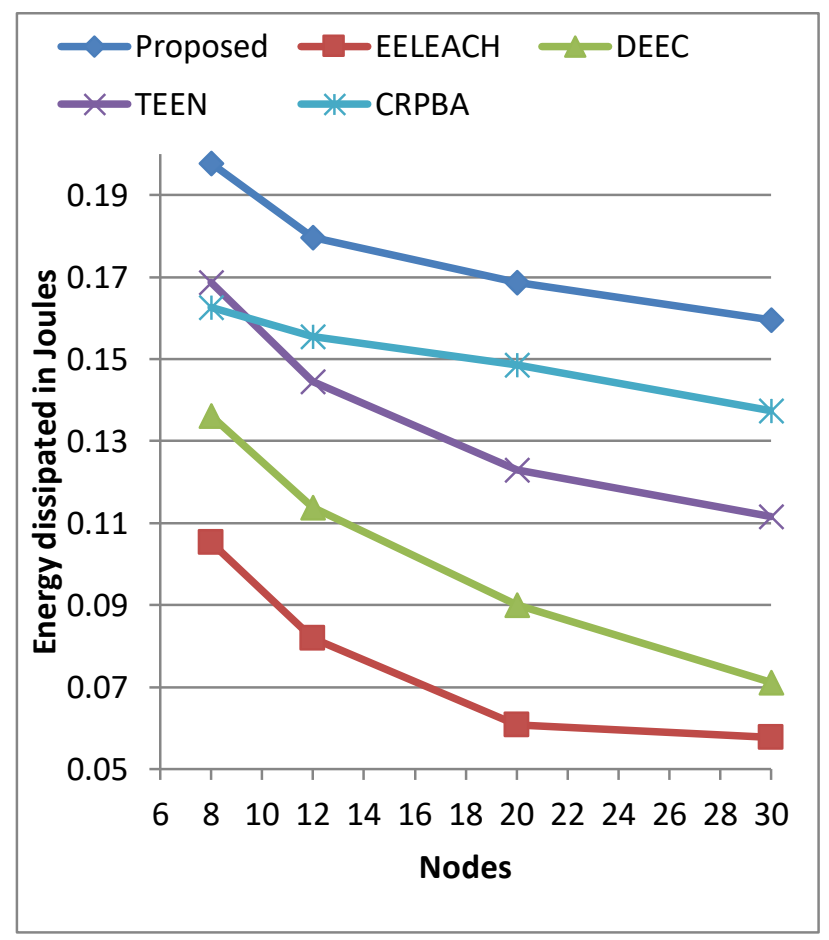

Fig. 4. Number of Nodes over Energy Dissipated.
TABLE III. PERFORMANCE EVALUATION OF AVERAGE REMAINING ENERGY FOR VARIOUS PROTOCOLS WITH 8, 12, 20 AND 30 NODES

\begin{tabular}{|l|l|l|l|l|l|}
\hline Nodes & \multicolumn{5}{|l|}{ Average Remaining Energy per Node (Joules) } \\
\hline & EELEACH & DEEC & TEEN & CRPBA & Proposed \\
\hline 8 & 0.1052 & 0.1361 & 0.1686 & 0.1627 & $\mathbf{0 . 1 9 7 7}$ \\
\hline 12 & 0.0820 & 0.1138 & 0.1445 & 0.1555 & $\mathbf{0 . 1 7 9 7}$ \\
\hline 20 & 0.0608 & 0.0901 & 0.1229 & 0.1485 & $\mathbf{0 . 1 6 8 8}$ \\
\hline 30 & 0.0577 & 0.0711 & 0.1115 & 0.1375 & $\mathbf{0 . 1 5 9 5}$ \\
\hline
\end{tabular}

\section{Throughput}

From Fig. 5 and Table IV, BS total numbers of packets reception will depend on how many rounds do the nodes are alive. The more time the alive nodes send more packets to base station and thus increasing the throughput.

Also, it is seen that; the proposed protocol throughput is better than EELEACH, DEEC, TEEN and ERPBA protocols.

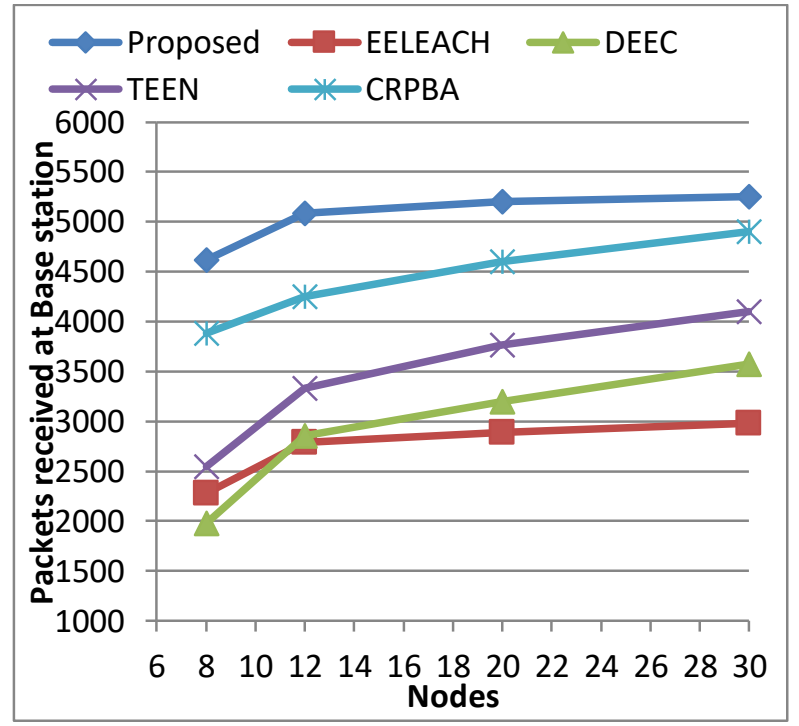

Fig. 5. Number of Nodes over Throughput.

TABLE IV. PERFORMANCE EVALUATION OF THROUGHPUT FOR VARIOUS PROTOCOLS WITH 8, 12, 20 AND 30 NODES

\begin{tabular}{|l|l|l|l|l|l|}
\hline Nodes & \multicolumn{5}{|l|}{ Throughput (packets for base station) } \\
\hline & EELEACH & DEEC & TEEN & CRPBA & Proposed \\
\hline 8 & 2280 & 1977 & 2545 & 3885 & $\mathbf{4 6 1 5}$ \\
\hline 12 & 2788 & 2857 & 3328 & 4254 & $\mathbf{5 0 8 5}$ \\
\hline 20 & 2890 & 3200 & 3770 & 4600 & $\mathbf{5 2 0 0}$ \\
\hline 30 & 2979 & 3570 & 4100 & 4900 & $\mathbf{5 2 5 7}$ \\
\hline
\end{tabular}

VI. CONCLUSION AND FUTURE WORK

Due to limited energy, complicated channel conditions, different body postures and data rates, the communication protocols developed for wireless sensor networks are not appropriate to WBANs. Hence, in this work, SNR based energy efficient communication protocol with a weightage function is developed and analyzed. At the initial phase, number of broadcast flooding's were reduced based on the distance from nodes and $\mathrm{CH}$, then energy saving paths were 
selected with respect to location of node and channel conditions. Finally, time slots were allocated for the nodes based on data priority of nodes. From the results, it is observed that developed protocol increases the network lifetime by $19.5 \%$, throughput is increased by $12.61 \%$ and average remaining energy by $57.21 \%$ for various nodes. The results show that the developed protocol is well suited for time critical applications by reducing the energy consumption while electing the cluster heads and data transmission. It would be appropriate and interesting to implement a prototype and evaluate the performance. Also, it might be challenging to see how it will work in small size of the network.

\section{REFERENCES}

[1] Nirupam Bajpai, John Biberman and Yingxin Ye, "ICTs and Public Health in the Context of COVID-19", CSD Working Paper Series: Towards a New Indian Model of Information and Communications Technology-Led Growth and Development, April 2020.

[2] Marcu, Ioana et al. "Arrowhead Technology for Digitalization and Automation Solution: Smart Cities and Smart Agriculture." Sensors (Basel, Switzerland) vol. 20,5 1464. 6 Mar. 2020.

[3] L. U. Khan, I. Yaqoob, N. H. Tran, S. M. A. Kazmi, T. N. Dang and C. S. Hong, "Edge Computing Enabled Smart Cities: A Comprehensive Survey," in IEEE Internet of Things Journal, April 2020.

[4] S. Talari, M. Shafie-khah, P. Siano, V. Loia, A. Tommasetti, and J. Catalão, "A review of smart cities based on the internet of things concept," Energies, vol. 10, no. 4, p. 421, Mar. 2017.

[5] Y. Qu, G. Zheng, H. Ma, X. Wang, B. Ji, and H. Wu, "A survey of routing protocols in WBAN for healthcare applications.," Sensors, vol. 19, no. 7, Apr. 2019.

[6] F. Rismanian, M. Hosseinzadeh, and S. Jabbehdari, "A Review of Stateof-the-Art on Wireless Body Area Networks," ijacsa, vol. 8, no. 11, 2017.

[7] M. Alrashidi and N. Nasri, "Wireless body area sensor networks for wearable health monitoring: technology trends and future research opportunities," ijacsa, vol. 12, no. 4, 2021.

[8] A. Sundar Raj and M. Chinnadurai, "Energy efficient routing algorithm in wireless body area networks for smart wearable patches," Comput. Commun., vol. 153, pp. 85-94, Mar. 2020.

[9] Y. Qu, G. Zheng, H. Wu, B. Ji, and H. Ma, "An Energy-Efficient Routing Protocol for Reliable Data Transmission in Wireless Body Area Networks.," Sensors, vol. 19, no. 19, Sep. 2019.

[10] B. Abidi, A. Jilbab, and E. H. Mohamed, "An energy efficiency routing protocol for wireless body area networks.," J. Med. Eng. Technol., vol. 42, no. 4, pp. 290-297, May 2018.
[11] N. Javaid, A. Ahmad, Q. Nadeem, M. Imran, and N. Haider, "iMSIMPLE: iMproved stable increased-throughput multi-hop link efficient routing protocol for Wireless Body Area Networks," Comput. Human Behav., vol. 51, pp. 1003-1011, Oct. 2015.

[12] A. Ahmad, N. Javaid, U. Qasim, M. Ishfaq, Z. A. Khan, and T. A. Alghamdi, "RE-ATTEMPT: A New Energy-Efficient Routing Protocol for Wireless Body Area Sensor Networks," International Journal of Distributed Sensor Networks, vol. 10, no. 4, p. 464010, Apr. 2014.

[13] N. Kaur and S. Singh, "Optimized cost effective and energy efficient routing protocol for wireless body area networks," Ad Hoc Netw., vol. 61, pp. 65-84, Jun. 2017.

[14] [4] R. A. Khan, Q. Xin, and N. Roshan, "RK-Energy Efficient Routing Protocol for Wireless Body Area Sensor Networks," Wireless Pers. Commun., vol. 116, no. 1, pp. 709-721, Jan. 2021.

[15] M. Anwar et al., "Green communication for wireless body area networks: energy aware link efficient routing approach.," Sensors, vol. 18, no. 10, Sep. 2018.

[16] M. Cicioğlu and A. Çalhan, "Energy-efficient and SDN-enabled routing algorithm for wireless body area networks," Comput. Commun., vol. 160, pp. 228-239, Jul. 2020.

[17] S. Ahmed, N. Javaid, M. Akbar, A. Iqbal, Z. A. Khan, and U. Qasim, "LAEEBA: link aware and energy efficient scheme for body area networks," in 2014 IEEE 28th International Conference on Advanced Information Networking and Applications, pp. 435-440, May 2014.

[18] N. Javaid, Z. Abbas, M. S. Fareed, Z. A. Khan, and N. Alrajeh, "MATTEMPT: A New Energy-Efficient Routing Protocol for Wireless Body Area Sensor Networks," Procedia Computer Science, vol. 19, pp. 224-231, 2013.

[19] A. Tauqir, N. Javaid, S. Akram, A. Rao, and S. N. Mohammad, "Distance Aware Relaying Energy-Efficient: DARE to Monitor Patients in Multi-hop Body Area Sensor Networks," in 2013 Eighth International Conference on Broadband and Wireless Computing, Communication and Applications, pp. 206-213, Oct. 2013.

[20] Q. Nadeem, N. Javaid, S. N. Mohammad, M. Y. Khan, S. Sarfraz, and M. Gull, "SIMPLE: Stable Increased-Throughput Multi-hop Protocol for Link Efficiency in Wireless Body Area Networks," in 2013 Eighth International Conference on Broadband and Wireless Computing, Communication and Applications, pp. 221-226, Oct. 2013.

[21] S. Ahmed et al., "Co-LAEEBA: Cooperative link aware and energy efficient protocol for wireless body area networks," Comput. Human Behav., vol. 51, pp. 1205-1215, Oct. 2015.

[22] L. Mao and Y. Zhang, "An energy-efficient LEACH algorithm for wireless sensor networks," in 2017 36th Chinese Control Conference (CCC), pp. 9005-9009, Jul. 2017.

[23] W. R. Heinzelman, A. Chandrakasan, and H. Balakrishnan, "Energyefficient communication protocol for wireless microsensor networks," in Proceedings of the 33rd Annual Hawaii International Conference on System Sciences, p. 10, 2000. 